\title{
MASS EXCHANGE VIA PULSATIONAL DUMPING FROM AN F SUPERGIANT
}

\author{
S. B. PARSONS \\ Astronomy Programs, Computer Sciences Corporation \\ Space Telescope Science Institute, 3700 San Martin Drive \\ Baltimore, MD 21218, U.S.A. \\ and \\ R. C. DEMPSEY and B. W. BOPP \\ Department of Physics and Astronomy, The University of Toledo \\ Toledo, $\mathrm{OH} 43606$, U.S.A.
}

\begin{abstract}
HD 207739 = V1914 Cyg, a tidally distorted F8 supergiant plus an obscured hot nondegenerate star, is presently in a state of episodic mass dumping onto an accretion shell around the hot component. Unrelated to the 141 day orbital cycle, the obscuration has a 50 day periodicity during which most of the UV flux diminishes rapidly, then gradually brightens. The more evolved yet less massive $\mathrm{F}$ supergiant is thus presumably shedding mass due to its pulsation.
\end{abstract}

\section{Introduction}

The binary star HD 207739, discovered to be interacting by Parsons, Holm, \& Kondo 1983, contains a luminous F8 primary and a partially obscured massive hot secondary. At the UV-bright epochs, the far-UV spectrum resembles a B1 giant with a few extra absorption features. At UV-faint epochs, the UV flux is reduced at most wavelengths by a factor of $2-3$; the very peculiar spectrum, with an apparent temperature of late B-type, resembles out-of-eclipse spectra of interacting systems such as SX Cas and TT Hya, with complex absorption features from warm circumstellar plasma at around $10000 \mathrm{~K}$.

Radial velocities of the F8 primary in the HD 207739 system demonstrate a well-defined nearly circular 140.78 day orbital period, with a mass function of 5.0 $\mathrm{M}_{\odot}$ (Griffin et al. 1990). The implication of the large mass function is that the hot star is more massive than the F-type "primary" (in visual light), and that the evolved F-type component must therefore have lost much mass since it left the main sequence. Preliminary analysis of a few UV lines attributed to a hot photosphere indicates a mass ratio of about 0.6 between the $\mathrm{F}$ star and the $\mathrm{B}$ star. This implies masses of about 14 and $23 \mathrm{M}_{\odot}$, respectively.

Photoelectric photometry, particularly by Bloomer 1984, Fernandes 1984, Szabados 1990, and Bopp (see Dempsey 1987) shows large ellipticity effects in the V light curve (a range of $0.26 \mathrm{mag}$ ) and irregular behavior in the $U$ magnitude. There is also variable polarization (Schutt et al. 1988). An analysis of the photometry yields an estimated orbital inclination of about $56^{\circ}$ and radius of the $\mathrm{F}$ star of about $92 \mathrm{R}_{\odot}$, about $60 \%$ of the formal Roche lobe radius.

Although the spectral classification of the primary is F8 II (Bidelman 1954), the radius implies a higher luminosity $\left(\mathrm{M}_{\text {bol }} \approx \mathrm{M}_{v} \approx-5.3\right)$ comparable to type Iab-Ib. With this luminosity and the derived difference of about $1.8 \mathrm{mag}$ between visual 
luminosity of the components at the least obscured phases, the B1 type leads to an estimated radius of around $9 R_{\odot}$ for the hot component.

Analysis of the composite energy distributions from far UV to near IR shows evidence of extra radiation in the mid UV for HD 207739 and at least several other related systems with strong $\mathrm{H} \alpha$ emission and peculiar UV spectra (see Parsons, Dempsey \& Bopp 1988; Dempsey et al. 1990). Such excess radiation can be accounted for by radiation from accretion disk material at about $10000 \mathrm{~K}$, similar to the inner boundary region of the disks around T Tau stars. The strong $\mathrm{H} \alpha$ emission also is indicative of a permanent disk around a relatively small mass gaining component (Peters 1988). In terms of visual light curves and obscuration of the hot components, HD 207739 and related systems are intermediate between classical Algol systems and Plavec's W Ser group.

\section{Ultraviolet Observations}

Far-UV and mid-UV spectra of HD 207739 have been obtained from time to time with the IUE Observatory since early 1982 . The epochs were generally sparse until a campaign in 1990 obtained observations at intervals of about 14 days over one entire orbital cycle.

For analysis, UV magnitudes representing fluxes averaged over $50 \AA$ bins were calculated. Standard IUE flux calibrations were applied, then sensitivity degradation corrections. The results (with or without degradation corrections) show real variations of more than one magnitude in some bins.

The spectral characteristics are correlated with flux level: UV spectra from different epochs and obscuration phases which have the same UV magnitudes are remarkably alike.

The few high dispersion far-UV observations show temperature variation of the absorbing plasma, in that spectra which look "earlier" in spectral type indicate a higher average ionization state. The spectra at lower flux levels exhibit prominent C II 1335 emission even at low dispersion.

The mid-UV spectrum of HD 207739 shows very strong $\mathrm{Mg}$ II emission and several Fe II emission and absorption features. High-dispersion observations reveal P Cygni profiles not only of the $\mathrm{Mg}$ II 2795, 2803 resonance doublet but also of $\mathrm{Fe}$ II transitions, notably multiplets 62 and 63 around $2750 \AA$.

\section{Analysis}

The ultraviolet behavior of the HD 207739 system has been very puzzling, since there is no real correlation of the flux variations with orbital phase. The observations in 1990 revealed that there might be a shorter periodicity to the variations. A period finding routine run on the $1450 \AA$ and $1650 \AA$ fluxes suggested a periodicity of 50.12 \pm 0.02 days.

Plots of all flux data against this period show a convincing correlation but a large amount of scatter. When the deviations from a mean slope are plotted against orbital phase, they show that the scatter is only large during orbital phases 0.0 0.5 , when the $\mathrm{F}$ supergiant is receding from the observer. By segregating the data 
between halves of the orbit, we find for orbital phases $0.5-1.0$ an extraordinary correlation between far-UV brightness and phase based on the 50.12 day period (Fig. 1).

The overall energy distributions have been analyzed with the tool developed by Parsons and Ake which provides trial-and-error fitting with combinations of standard star intrinsic colors (and optionally a Planck spectrum) and a standard interstellar extinction curve. With $\mathrm{E}(\mathrm{B}-\mathrm{V})=0.30$ and black body temperatures in the range 8000 to $12000 \mathrm{~K}$, it is possible to fit the entire binary system's radiation from far-UV to near-IR.

The Planck component's mid-UV flux is fairly well correlated with obscuration phase, but also has a moderate orbital phase dependence. This radiation component is brighter on average during orbital phases $0.0-0.5$, when we presumably see the opposite side of the disk from the impact region.

\section{Discussion and Conclusions}

We hypothesize that the 50.1 day cycle is due to pulsation of the F supergiant. The mass exchange in the system occurs mainly during a brief portion of each cycle, causing the UV flux to drop abruptly as the material first reaches the vicinity of the hot star and causes maximum obscuration. The obscuration declines over the rest of the cycle but never quite fully reveals the underlying star. The effect is clearest on the side of the accretion "disk" which receives the material directly. Maximum obscuration is also seen on the other side at the same phase, but the dissipation/accretion of the matter appears not to be as regular as on the impact side.

The accretion "disk" must have considerable vertical extent relative to the small hot star, in order to show obscuration effects at the $34^{\circ}$ tilt of the orbit plane to the line of sight. A large vertical extent is suggested also by Plavec 1988 for the TT Hya system. The dynamics of pulsationally propelled mass loss would cause much of the mass transfer to occur out of the orbit plane.

The 50 day period is quite long compared with classical Cepheids. The radius of $92 \mathrm{R}_{\odot}$ derived for HD 207739 would correspond to a Cepheid period of about 14.4 days. According to theoretical work by Lovy et al. 1984 on supergiant pulsation, the majority of non-Cepheid supergiant stars exceed the predictions (about 16 days in this case) by a factor of 2-3 due to non-radial pulsations or to more advanced evolution with greater mass loss than assumed in the models. We therefore see no problem with the relative length of the 50 day period, since there has evidently been substantial mass loss from the $\mathrm{F}$ primary in this system, and since the effective gravity and mean density is undoubtedly reduced in a close binary system relative to single stars.

Pulsational mass dumping is not a new concept. Kalv 1979, for example, suggests that the G giant in the RX Cas system is pulsating; because it fills its Roche surface, it does not vary significantly in light itself but causes the A star to vary because of the changing obscuration.

Every 50 days, HD 207739 becomes a semi-detached system for a brief interval. Thus it might be classified as an episodic long-period Algol binary. 


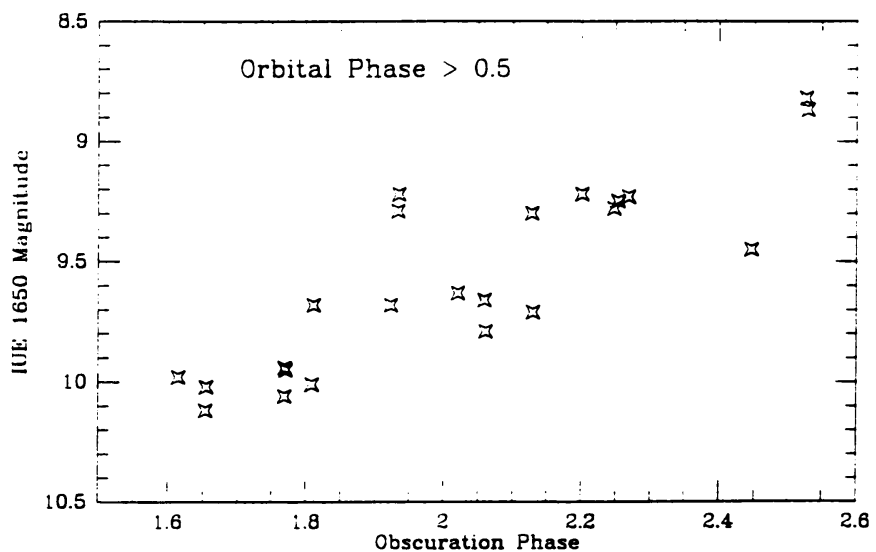

Fig. 1. Far-UV flux as function of "obscuration" period of $\mathbf{5 0 . 1 2}$ days. The data displayed are only for orbital phases when the hot star is receding. When the probable impact area for matter from the $\mathrm{F}$ star is toward the observer, a correlation is very clear.

\section{Acknowledgements}

SBP and BWB are Guest Observers with the International Ultraviolet Explorer Satellite. This analysis has been performed primarily at the IUE Regional Data Analysis Facility at Goddard Space Flight Center. SBP's research is supported by NASA contract NAS 5-28749 to the Computer Sciences Corporation. RCD is now at Joint Inst. for Lab. Astrophysics, University of Colorado, Boulder, CO 80309-0440, U.S.A.

\section{References}

Bidelman, W. P. 1954, ApJS, 1, 175

Bloomer, R. H. 1984, BAAS, 16, 913

Dempsey, R. C. 1987, Master of Science thesis, The University of Toledo

Dempsey, R. C., Parsons, S. B., Bopp, B. W., \& Fekel, F. C. 1990, PASP, 102, 312

Fernandes, M. 1984, IBVS, No. 2536

Griffin, R. F., Parsons, S. B., Dempsey, R., \& Bopp, B. W., 1990, PASP, 102, 535

Kalv, P. 1979, Tartu Astron Obs Teated, No. 58, 3

Lovy, D., Maeder, A., Noëls, A., \& Gabriel, M. 1984, A\&A, 133, 307

Parsons, S. B., Dempsey, R. C., \& Bopp, B. W. 1988, in A Decade of UV Astronomy with IUE, ed. E. J. Rolfe (ESA Pub. SP-281), Vol. 1, p. 225

Parsons, S. B., Holm, A. V., \& Kondo, Y. 1983, ApJ (Letters), 264, L19

Peters, G. J. 1989, Space Sci Rev, 50, 9

Plavec, M. J. 1988, AJ, 96, 755

Schutt, R. L. et al. 1988, BAAS, 20, 736

Szabados, L. 1990, A\&A, 232, 381 